Title: Enabling practitioners working with young people who self-harm

Foster C., Birch L., Allen S., Rayner G. (2014)

In Press: Journal of Mental Health Training and Education Practice

\begin{abstract}
Purpose: This paper outlines a UnitedKingdom based interdisciplinary workforce development project that had the aim of improving service delivery for children and young people who self-harm or are feeling suicidal.
\end{abstract}

Approach: This innovative practice-higher-education partnershiputilised an iterative consultation process to establish the local workforce need and then facilitated the systematic synthesis and presentation of evidence-based clinical guidelines in a practical format, for staff working directly with young people who self-harm in nonmental health settings.

Outcomes: The development, content and structure of this contextualised resourceis presented, along with emerging outcomes and learning from the team.It is anticipated that this may also be a useful strategy and resource for other teams in other areas and is intended to provide a template that can be adapted by other localities to meet the specific needs of their own workforce.

Practical Implications: The paper demonstrates how higher education-practice partnershipscan make clinical guidelines and research evidence in a field often thought of as highly specialist,accessible to all staff. It also shows a process of liaison and enhanced understanding across universal/specialist mental health service thresholds.

Originality/Value: This paperdemonstrates how collaborative partnerships can work to bridge the gap between evidence-based guidelinesand their implementation in practice, through innovative multi-agency initiatives.

Keywords: Self-harm, suicide, children, young people, interdisciplinary, workforce development, risk assessment, stakeholder engagement, mental health, adolescent

Paper Type: case study

\title{
Background
}

Self-harm and suicidal acts amongst children and young people are a high priority for health, social care, education and specialist children's mental health services alike. Whilst exact prevalence of these behaviours is very difficult to establish, some evidence points to rates in the UK being the highest in Europe and possibly on the increase (Hawton et al, 2012; Madge et al, 2008). Prevalence across the full age range of children and adolescents has been estimated at $10 \%$ (Hawton et al, 2012). Onset of self-harm across the life course is most 
highly associated with adolescence (Moran et al, 2012), and whilst rates vary across this time (Mental Health Foundation: Camelot Foundation, 2006) it is fair to say that self harm is an issue that affects young men and women in equal but qualitatively different ways. Research evidence continues to suggest that up to $30 \%$ of children and young people consider self-harm at some point in their lives and that within those who go on to actually hurt themselves, only around $12 \%$ seek professional help (Madge et al, 2008, Nock, 2010).

These findings combined with learning from Serious Case Reviews (Cumbria LSCB, 2014; Pearson, 2008), highlight a number of apparent paradoxes. Self-harm is both a universal issue affecting many young people in the context of ordinary developmental processes, and for a small number is also highly perilous, correlated with child protection issues, a higher risk of suicide and poor experience of helping services and stigma. Self-harm is conceptualised as a distinct phenomenon from suicide serving different functions, whilst at the same time statistically linked to an increased risk of suicide across the life course (for a full review of the literature see: Foster, Rayner and Allen, 2012).

Although much is now known and understood about the underlying causes, precipitants, and needs of children and young people who self-harm, from a workforce development and service delivery perspective it remains a very challenging and complex issue. This is largely because this group who harm themselves or have thoughts of self-harm is heterogeneous. Young people's needs and presenting risks are diverse, requiring different responses, from different constellations of services, at different times (Hawton et al, 2012; MFH: CF, 2006). Thispopulation of children and young people who self-harm can be thought of as being broadly constituted of those young people:

- Who are suicidal

- For whom self-harm is a marker of significant safeguarding issues

- Who repeatedly self-injure (often in very risky but non-fatal ways) in the context of complex intra and interpersonal relationship difficulties

- Whose self-harm is a linked to psychiatric illness or disorder (e.g. depression or anxiety)

- For who it is a (often one-off) stress response to being overwhelmed by developmentally ordinary psychosocial stressors, most helpfully responded to by any professional with whom they have an existing relationship.

Although this last group is by far the largest component of the identified population of young people who self-harm (Hawton et al, 2012), the known relationship between self-harm and an increased statistical risk of suicide (NICE, 2011) can understandably make many professionals in children's services other than specialist child and adolescent mental health (referred to as universal from this point), anxious to intervene. This relates to professional fears of making things worse or of acting outside of the scope of one's knowledge and skills. This has been observed to lead to workers responding to all cases of self-harm by referring onto to specialist child and adolescent mental health services (CAMHS), and declining to intervene themselves. However, the established epidemiological risk factors highlight that self-harm and suicidality in children and young people is most often a psychosocial issue, 
often requiring a non-psychiatric, pragmatic resolution of the precipitants and triggers. Examples include interpersonal stress within key relationships, the experience of bullying, discrimination or maltreatment or social adversity (Webb, 2002, Crowley et al, 2003). This indicates that there is a significant role for the universal children's workforce in supporting and enabling young people to work through and resolve the contributing stressors. However, in order to do this, an accessible framework of evidence based guidance and support that addresses the workforce's legitimate concerns is required to make this a reality.

The evidence-based practitioner resource outlined in this paper is explicitly designed to address these concerns in an innovative format. Whilst there have been materials previously published that aim to draw together evidence and guidelines (for examples see: Royal College Psychiatry, 2010; Catlkedge et al, 2012), these have tended to be descriptive evidence summaries stating 'what' is required, without guidance about 'how' to achieve it. Previously published materials of this kind have also been largely aimed at specific unidisciplinary groups within the workforce. This paper describes Knowsley Metropolitan Borough Council endeavour to develop an evidence based resource suitable for multidisciplinary workers across the universal children's workforce to support them with 'how' to achieve 'what' is required.

Development of this resource formed one strand of a wider collaborative self-harm service development project between the commissioners and providers of children's servicesand a local university. The project also sought to meaningfully engage young people and their families as stakeholders in the process. This wider project is briefly outlined to provide context, before a detailed account of how the practitioner resource was developed

\section{Local Context}

A local needs assessment confirmed that Knowsley has relatively low levels of reported selfharm, being 8th lowest across North West England authorities for emergency admissions per 100,000 population for 2007/08 and 2009/10 (Holford, 2012), and relatively low levels of suicide. Anecdotally however, practitioners across the children's workforce were reporting high levels of identified or self-reported self-harm, and between 2008 and 2012 there had been 4 suspected child suicides and one suspected suicide for a young adult in transition (Holford, 2012). Within the local needs assessment, a number of examples of good practice were identified; however, there was limited evidence of coordinated responses to young people whose self-injurious or suicidal behaviour and intentions caused concern. Children's service providers acted with very good intentions but often in isolation of each other and without access to relevant information concerning the young person, or knowledge of other services. In response to these findings a 12 month service development project was agreed

\section{Overview of the Service Development Project}

$\underline{\text { Aims }}$

To understand what was happening in the locality/community; the evidence; young people's, family's and practitioner experience. 
To use this information to inform:

- Health promotion,prevention, and safeguarding strategies in the widest sense (e.g. bullying, domestic violence strategies), not just in relation to self harm and suicide

- Strengthening knowledge, skills and confidence within the universal children's workforce - so that most young people can be helped in the context in which they usually live and learn, by people they know

- Care pathways to ensure young people get access to the right specialist mental health services when they need them

- Strengthening existing, and building new, multi-agency ways of working for young people at high risk, and whose complex needs put them on the margins of multiple service providers

The outputs agreed to achieve these aspirations are illustrated in Figure 1.

This paper focuses on development of a practical evidence-based practitioner resource to aid assessment and decision making, which was central to the achievement of project aims $2 \& 3$. The contribution of service providers to the design of the resource also enabled them to work together to develop the operational protocol for multi-agency responses to young people identified as being at high risk and/or having complex needs (project aim 4).

\section{Creating a Commissioning-Higher Education-Practice-Community Partnership}

A strength of the project was that from the outset it had representation from practitioners across the Knowsley children's workforce from health, education, social care, voluntary, youth, police, youth justice and advocacy services, working with our Knowsley children and young people, who were committed to and engaged in the process.

However, early in the development of the required scheme of work, there was recognition of limited capacity and resource within the locality for some of the tasks identified, i.e. finding, synthesising and differentiating the evidence base for different audiences, building capacity for reflective learning methods and developing facilitators to deliver these. It was on this basis that a partnership with a local university was sought by the locality children's service commissioners. In addition, by commissioning a university to undertake some aspects of the project specification, within an agreed contract of expectations and outcomes, work could be completed within the given timeframe.

The University of Salford has a well-established body of clinical, teaching, workforce development and research expertise specifically in relation to self-harm and child and adolescent mental health. This included long standing Post-qualifying and Postgraduate education programmes in both of these subject areas. Within the project team all the university academics were still engaged in clinical practice relevant to these fields alongside their academic roles.This contributed to perceived credibility in the eyes of the local children's workforce and an ability to understand the landscape within which frontline practitioners were operating. 
Two project groups were formed for the purpose of governing and implementing the project. A Steering Group made up of managers and heads of service, and an Implementation Group predominantly made up of front line workers. Both groups were representative of the spectrum of services for children and young people across health, education, local authority and the voluntary sector domains within the locality of Knowsley. The link between the two groups was maintained through membership of the project manager and of the university academics in both groups. Young people affected by the issue of self-harm were engaged as advisors and consultants to the project through locality participation groups, which also had representation on the steering group.Clear leadership, project management, governance and accountability processes were provided by the locality children's services commissioners, aided by assignment of the project manager.

In order to promote genuine collaboration and influence, rather than feeling subject to top down impositions, an iterative process of both face-to-face and electronic consultation and feedback in relation to all outputs of the project, between stakeholders and the university was coordinated by the project manager.

\section{Practitioner Resource Development}

\section{Method}

Establishing stakeholder agreementin the production of the practitioner resource

An important tension had to be resolved in the initial phases of strategic level project management before any development work could begin. Initially,it was agreed that a resource for universal workforce practitioners to aid decision-making processes in relation to young people who disclose self-harm would be developed by the university. However, in a later document produced by the project commissioners describing the agreed outputs, an understandable but unachievable underlying wish that the university would identify the 'right' risk assessment tool to accurately predict and prevent future suicides was expressed. This was despite clear evidence and guidance being available on the limits of such checklists (NICE 2011; RCPsych, 2010; Littlechild and Hawley, 2010).

Discussion of the relative merits of these two different positions in light of the available evidence led to agreement between stakeholders regarding the intended purpose of the resource as follows:

- To create an easy-to-use, evidence based resource with a transparent language and a set of principles shared across all tiers of the workforce, facilitating dialogue between universal children's service worker and specialist CAMHS practitioners

- To act as an in-situ tool for leading non-mental health practitioners through the appropriate responses, if a young person discloses actual or thoughts of selfharm or suicide. Thereby helping with the triaging and decision making process. 


\section{Content}

As per Figure 1, a comprehensive practice-focused literature review was completed as part of the wider project outputs. This was undertaken utilising a systematised search and critical review method, as outlined by Grant and Booth (2009). This method aims for an exhaustive and comprehensive searching process, in which findings from diverse sources are evaluated according to their contribution to the issue under interrogation and are subject to critical analysis and synthesised to inform conceptual innovation. This review method is indicated for production of best evidence synthesis in health subject fields (Grant and Booth, 2009). From this the evidence-based content for the resource was identified.

A series of four face to face consultation events were held across the locality to identify the needs and concerns of practitioners regarding working with young people who hurt themselves or feel suicidal. All staff in the universal and specialist children's mental health workforce were invited. The events were attended by staff from health, social care, nonstatutory support agencies, local authority departments, primary, secondary and further education, youth offending services and the police. The consultation events were facilitated by university staff, in order to be non-partisan, utilising a focus group method. Transcripts from the consultation meetings were subject to thematic analysis in accordance with the method outlined by Braun and Clarke (2006), enabling individual contributions to be anonymised and key concerns and priorities identified.

The outcomes of the consultation process were utilised to prioritise and organise the required content extracted from the literature review, in order to respond to the expressed needs of the local workforce. Findings from the literature review, the consultation process, and proposed content for the resource were presented to the steering and implementation group and to consultation attendees for further comment and feedback.

\section{Design}

Priorities identified by staff involved in the consultation process were that the resource be portable, quick reference and in a form that could be utilised when with children and young people. On this basis, digital and web-based material was overwhelmingly declined, as most practitioners reported that they did not have IT access at the points of direct contact with children. A product that could easily be carried in a diary, but that was electronically available for re-printing, updating and sharing with others as needed was favoured.

Agreed content was subject to a design and cyclical refinement process: The university's design and reprographics team's expertise was utilised to optimise use of space and maximise logical flow, clarity and coherency of the content. The steering group, implementation group and consultation attendees became an expert panel to provide feedback on whether the design team's intentions had been achieved in practice, and on overall acceptability and practical usability. This was repeated utilising a structured consensus-orientated decision making approach (Hartnett, 2010) until all stakeholders felt that tensions and disagreements about the material had been sufficiently reconciled to meet their needs. As a concrete illustration of this reconciliation process, the final resource was created on double sided A3 format to enable all of the required detail to be included, and formatted to fold like a map to A6, in order to genuinely meet the brief of being 'pocket' sized. 


\section{$\underline{\text { Outcomes }}$}

Synthesis of evidence to inform the resource content

In order to produce this resource the following evidence based principles for good practice were identified from the data synthesis of the literature review:

Suicide and self-harm are multi-determined acts in which a complex range of experiences come together in a way that is unique for the individual and that particular occasion (RCPsych., 2010; Underwood, 2009; Hawton \& James, 2005). As a result, responding helpfully at any level requires a therapeutic rapport with a young person in order to understand and respond to their particular unique combination of factors and subjective experience. Establishing a shared understanding of the function and meaning of self-harm with a young person at each particular time constitutes the foundation of all assessment, response and intervention recommendations (NICE, 2011, 2004; Nock, 2010; Skegg, 2005). This needs to be embedded in all local policy initiatives and priorities and into the philosophy of care within the local workforce. Risk assessment should be based on understanding of individual circumstances and intent, utilising a narrative approach, not through use of actuarial risk-assessment checklists (RCPsych, 2010; NICE, 2004 \& 2011; Appleby et al, 2012).

Risk reduction, risk management and recovery pivots on development of therapeutic trusting relationships aimed at shared understanding, reducing shame and stigma and making young people collaborators in their own care. It must address underlying problems and wider psychosocial and safeguarding risks, engaging and supporting young people as a helpful and thoughtful other who can assist with problem solving (Hawton et al, 2012; Hill, Castellanos et al, 2011; NICE, 2011; Pryjmachuk and Trainor, 2010; MHF:CF, 2006).

Development of workforce competency in relation to self-harm should utilise a whole system approach across disciplines, departments and agencies (Appleby et al 2012; Skegg 2005). The most recent national clinical guidelines (NICE, 2011) prioritise establishing the value of empathic listening and understanding in the minds of practitioners, not just as conditions for positive experience, but as effective interventions in themselves that are empirically correlated to risk reduction and recovery. Self-harm has been shown to stir up feelings of fear, anxiety, anger and helplessness in the helper (Walsh, 2007; Cooke and James, 2009; Crawford et al, 2003), mirroring the feelings identified by those who self-injure (Rayner et al, 2005). To counter this, self-compassion has been identified as an essential foundation to being helpful to others experiencing self-harm and mental distress (Rayner 2012; Gilbert, 2005).

This resonates strongly with recommendations from the National Inquiry into Self Harm Amongst Young People (MHF:CF, 2006), for building confidence in the workforce through stressing that competent practice with young people who self-harm is based on a reconnection with core skills and values of all caring professions and providing responses that are rooted in these.

Both the National CAMHS Support Service (2011) and the Findings of the National Inquiry (MHF: CF, 2006) have identified minimum levels of knowledge and skill for all those working with children and young people. In summary these are: 
- Understanding what self-harm is, the underlying reasons for it and the functions it can serve for young people

- How to respond to disclosures of self-harm, helpfully, sensitively and appropriately

- A clear understanding of the legal framework in relation to consent, competence, capacity and safeguarding

- Knowledge of what other support and services are available.

- Ability to contribute to challenging societal and professional attitudes that create stigma and exacerbate the difficulties of young people who self-harm

In addition, the Royal College of Psychiatry (2010) highlights the value of non-mental health staff knowing indicative signs and symptoms of common mental health disorders, translated into simple language that any professional can feel confident to ask about.

The evidence regarding the challenges of engaging non-mental health practitioners in this work and the means by which these can be overcome was also reviewed and incorporated into the final product design. The importance of having a theoretical understanding of the symbolic, emotional, psychological and physical functions and meaning of self-harm has been stressed by multiple authors as pivotal in reducing the tendency to over-focus on physical manifestation of self-harm and in challenging staff assumptions regarding controllability; which have been shown to underpin negative attitudes towards individuals (Cook \& James, 2009; Mackay \& Barrowclough, 2005). Being supported to work out 'when' and knowing 'how' to refer on to specialist mental health services have been shown to help with feelings of practitioner helplessness, which in turn can protect against hostile attitudes within professionals toward those who self-harm (Cook \& James, 2009; Crawford 2003). This was reflected in the content and structure of the resource produced.

\section{Thematic Analysis of Workforce Consultations}

Individually held definitions of self-harm and what should be considered as self-harm varied significantly amongst consultees, resulting in a request for any resource to begin with a locally adopted definition.

Key concerns raised by practitioners centred on fears regarding their own lack of understanding, that the skills they used in their everyday work were not transferable to this issue, and that engaging with young people around the issue of self-harm triggered worries about making things worse. These fears underpinned a commonly expressed belief that all young people who self-harm or express thoughts of self-harm should be referred to specialist mental health services. There was expressed dissonance between these views and first hand experiences of the local tier 3 CAMHS service not accepting all referrals for young people who had self-harmed, leading to feelings of frustration and confusion about seemingly opaque criteria for referral to specialist mental health services.

Despite concerns raised there was recognition amongst nearly all attendees that they might be the first point of contact or disclosure for a young person. In line with this, requests for content on the resource centred on wanting to develop confidence so that if they were the first person a young person confided in, they could listen and talk to the young person without making it worse. This comprised of: 
- Myth busting - many attendees identified beliefs that all self-harm is a suicide attempt, that it is always a sign of mental illness or personality disorder and that asking about suicide will make young people who had not previously considered ending their life suicidal. Attendees were aware that these beliefs had developed in lieu of access to factual information about self-harm and suicide.

- When to refer on and when they could be helpful within their role.

- Guidance on decision making - what to do next after a young person has disclosed their self-harm.

- Help to identify those who are suicidal or might need specialist referral. In particular, what questions to ask and how to ask them. Including requests for explicit examples of how to phrase questions about a young person's intent.

- Access to advice, credible resources and self-help materials and information about other services was identified as a significant mitigator in whether they would feel able to engage with a young person safely and confidently or not.

A strong emergent theme in the consultation process was participants' requirement for supervision that attended to the emotional content and impact of this work, rather than purely focused on performance management. The supervisor was also required to have knowledge of evidence based practice in relation to self-harm and support an approach that is built upon realistic expectations of risk reduction, rather than risk extinction.

Concerns amongst the specialist CAMHS practitioners who attended the consultation events were that all young people who expressed thoughts of self-harm were being referred to specialist services without triage of severity or attempts to understand the context of the issue for the young person; that once a referral had been accepted universal staff would take flight from contributing to the young person's ongoing care.

Concurrently, some representatives from both CAMHS and universal children's services expressed worries that the resource development represented on the part of children's service commissioners, an indirect means of asking the latter to take over responsibility for specialist mental health assessment.

These seemingly dialectically opposed points, reflecting the all-or-nothing positions that the risks associated with self-harm can evoke in professionals (Rayner et al, 2005), strongly informed the structure and flow of the final resource. In that, it attempted to model a pathway in which all those working with children can be helpful and efficacious, whilst understanding that there are a sub-group of young people who require specialist help. This was specifically addressed within the resource by providing a process for identifying these young people and for communicating this effectively to specialist services. 


\section{Final Practitioner Resource Content and Structure}

Figure 2 shows a schematic summary of the resource content and its organisation. This is intended to provide a template that can be utilised and adapted by other localities to meet the specific needs of their own workforce.

Figure 2: Schematic illustrating resource content and structure

\section{Implementation}

The final resource was disseminated to the workforce at a formal launch event hosted by Knowsley Children's Safeguarding Board. Information about the wider service development project and the resource development process was provided along with interactive workshops to outline the intended use of the resource, address practitioner questions and provide information about the additional support mechanisms put in place to enable implementation.

The resource was linked to development of a self-harm awareness-raising training syllabus for the entire children's workforce. This was made available to all staff, followed up by access to facilitated multi-agency reflective learning sets for a period of 4 months, to help practitioners embed the new guidance and their learning into their everyday practice. This approach was adopted in line with the evidence that supports access to reflective learning strategies when working with adolescents and self-harm; to enable practitioners to implement new knowledge, and to manage the emotional impact of the work in their subsequent interaction with the young person (RCPsych, 2010; Briggs et al, 2009; Rayner et al, 2005).

Critical Reflection - lessons learnt from the process:

The process outlined for undertaking this project was as much a product of pragmatic problem solving as it was of strategic and intentional design. As such, undertaking a process of critical reflection in order to identify lessons learnt and their implications has been an important part of the process. This was facilitated with the use of Driscoll's (2007) model, 'What? So What? Now What?'

There are a number of alternative approaches that could have been taken. One option would have been to secure the time of several practitioners within the authority, dedicated to the development and delivery of the products outlined in the specification. However, the short timeframe in which we had to deliver this project successfully prevented this. Relying on practitioners to find time away from their primary roles to undertake workforce development initiatives, has been demonstrated to significantly delay project progress (Blackie, 2004) 
The benefit of working alongside academic partners in this project was their expertise of clinical research and practice but also the ability to deliver within the timescales and access to the wider university resources (e.g. product design). However, it was also vital that clinicians were identified as a source of clinical expertise to the higher education team, to ensure both 'buy-in' to the project and effective delivery (Blackie 2004). We have identified that meeting together on a regular face-to-face basis through the implementation group, as essential to this.

Hudson (2002) highlighted that successful inter-professional working in health and social care is down to focusing on the development of inter-professional relationships rather than inter-organisational policies. Differences of perspective in relation to professional culture, and respective misunderstandings of each other's professional identity has been shown to significantly impact upon the success of health and social care partnerships (Brady, 2013; Hudson 2002). Having both the Steering Group and Implementation Group was critical to the outcome of the project. Having time and space built into the scheme of work, in which partners could come together to understand each other's perspectives and reconcile any tensions, rather than assuming policy alone would bring about uniformity, has been pivotal to the cooperation and openness across agency thresholds and boundaries observed as the project progressed.

In relation to managing the inter-professional and interagency requirements of the product and the need to differentiate the evidence based content to meet these needs, the two groups allowed us to be agile about how we developed the practitioner resource, taking into account the views of staff that would eventually use the tool, but ensuring we met the strategic brief which we had set out to deliver for the wider Knowsley workforce. That said, reflecting on our learning has also helped us recognise a significant limitation in the way in which the respective functions of these two groups were managed:

By far the biggest tension that emerged in the design of the practitioner resource was that of language. It was important that we developed tools and resources within the project that catered for all sectors and levels of expertise and it was necessary to keep language simple and straight-forward, but still accurately reflective of the evidence from which it was drawn. The subject of self-harm raises language not all practitioners are comfortable with. Combine that with an academic vernacular which has the potential to challenge and undermine trust between practice and academic institutions (Imaginative Curriculum Network, 2006) and it makes for a significant process of feedback and modification.

In fact, gaining consensus regarding the overall structure and content of the resource was relatively easy and swift. In contrast, agreeing terms and striking the balance between trying to produce something that was rich enough in detail to be useful, whilst trying to ensure we did not include too many opinions at the expense of clarity and usability is one we struggled 
with. In retrospect, we have identified that this was because assumptions about the relative weight given to each group's right of comment regarding particular aspects of the resource had not been explicitly defined at the outset. Whilst it made sense for the steering group's focus to have been limited to the compatibility of the resource content with existing strategic and organisational policy frameworks, and the practice implementation group's focus to be on in-situ practical usability, this was never explicitly laid out. Furthermore, final sign off of all phases of the project rested with the steering group. As a result, the process of agreeing the finer detail of specific words, phrases and semantics was much more involved, requiring multiple cycles of painstaking and patient consultation, feedback, modification and reconsulting, brokered by the project manager. The importance of going beyond the establishment of individual group terms of reference, in order to gain cross-group agreement at the outset in relation to operational terms and the hierarchy of decision makingin relation to different aspects of the resource, is a lesson that we will take into any future collaborations of this kind.

Inter-agency politics in a time of austerity and a perceived increase in service user demand, also contributed to the complexity of developing working partnerships (Brady, 2013). Colleagues appeared to be jostling for a position in relation to where their organisation featured within the resource or in relation to perceived/implied statements of their relative specialism within the field.

Whilst localcommissioners are clearly tasked with seeking agency integration and collaboration for patient benefit, in the UK the Health and Social Care Bill (2012) now also explicitly demands the fostering of competition, with some commentators observing that competition rather than collaboration is the default setting within the legislative framework (Hudson, 2013). Competition is noted to promote selfinterested rather than public interested behaviour and low rather than high trust relationships with other agencies. This is compounded by the observation that collaboration can directly threaten the ability of an agency to perform successfully within a competitive environment, due to loss of autonomy, and requirement to invest finite resources in a process with unclear and untested outcomes (Ranade and Hudson, 2003).

Although clearly unintentional, these issues meant that it was difficult to foster the ambition of a culture of shared responsibility and endeavour. In our analysis, two components of the project implementation contributed positively to overcoming these barriers. Firstly, engaging practitioners in the establishment of clearly agreed common purpose and intended outcomes at the very outset of the project (Brady, 2013). Stakeholder commitment to the essential aim (improved service quality and experience for young people) meant that individuals were able to show persistence and forbearance in putting up with individual losses or tolerating perceived attacks upon their professional identity and purpose for the sake of getting the resource right. 
Secondly, the importance of a non-partisan project manager in reconciling or finding a good enough resolution to the ongoing tensions we have outlined, and subsequent completion of the project, cannot be overstated. Not being aligned to any one agency, but only ensuring completion of the agreed tasks within the agreed timeframe, enabled relationships with agencies to be forged that were free of competition or suspicion of underlying agendas. Such boundary spanning roles are noted as significant in interagency partnership success, although their essential characteristics are not well researched (Ranade and Hudson, 2003). In this instance, providing a single point of contact for all, with a focus on facilitating communication, consensus building and pragmatic solution identification, and being able to mitigate and mediate when tensions did arise, were critical to the project's success

\section{Early Indicators of the Resource's Impact in Practice}

- High uptake of awareness raising training and reflective learning sets across the locality's children's workforce

- Evaluation of the impact of the multi-agency reflective learning sets has led to a number of agencies attending more specifically to the emotional support needs of their staff when working with self-harm during supervision.

- Increased engagement between CAMHS and universal children's services, including a commitment to strengthening of the CAMHS consultation and advice service.

- Increased engagement of children's social care services in the development of the cross-agency pathway for young people with complex needs at high risk of self-harm or suicide - an area traditionally understood within the locality as the exclusive domain of health services.

- Communication received from Public Health England (An executive agency of the UK Department of Health) of their intent to include the project as an example of good practice in a cross-government suicide prevention strategy for England.

- Positive anecdotal feedback received from workers using the resource in practice.

- Commitment of Knowsley Children's Safeguarding Board to continue implementing the recommendations and action plan developed from the literature review.

- Service commissioners have shown an increase in commitment to supporting children and young people who self-harm, in line with the service development project recommendations, evidenced in locality service plans. 


\section{Concluding remarks}

The passion and commitment that we experienced from professionals, service users and their carers across the Knowsley workforce during the delivery of this project was exemplary and our grateful thanks go to all those involved. Having consistent representation from partners with a wealth of experience working in all different sectors is crucial to the delivery of projects and resources of this nature. Feedback received from professionals using the resource in practice to date, has been positive and constructive. Next steps are to maintain current momentum and to systematically evaluate the usefulness and impact of the resource and the outcomes of the wider project.

\section{References}

Appleby L., Kapur N. Shaw J, Et al (2012) Report of the National Confidential Inquiry into Suicide and Homicide by People with Mental IIIness: Annual Report: England, Wales, Scotland, and Northern Ireland. TSO The Stationary Office

Blackie, P. (2004) Collaboration \& Partnership: An example from Greater Manchester, Forward, 3, 18-20.

Brady M (2013) The nature of health and social care partnerships. Nursing Management, 19(9), 30-35.

Braun, V., and Clarke, V. (2006). Using thematic analysis in psychology. Qualitative research in psychology, 3(2), 77-101.

Briggs, Stephen (2009) Risks and opportunities in adolescence: Understanding adolescent mental health difficulties. Journal of Social Work Practice, 23 (1), 49-

64.

Catledge C., Scharer K., Fuller S. (2012) Self Harm in Adolescents and Young Adults. Journal for Nurse Practitioners, 8(4), 299-305

Cooke, E. and James, V. (2009) A self-harm training needs assessment of school nurses. Journal of Child Health Care, 13, 260-274.

Crawford T., Geraghty W., Street K., Simonoff E. (2003). Staff knowledge and attitudes towards deliberate self-harm in adolescents. Journal ofAdolescence, 26 (5), 623-633.

Cumbria Local Safeguarding Children Board(2014) Serious case review: Child J: final report. Published by Cumbria Local Safeguarding Children Board (2014). Accessed at:www.cumbrialscb.com/eLibrary/Content/Internet/537/6683/6687/6700/4182185614.pdf 
Driscoll, J. (2007) Practising Clinical Supervision: A Reflective Approach for Healthcare Professionals. 2nd ed. Edinburgh: Bailliere Tindall Elsevier

Foster C., Rayner G., Allen S. (2012) Self harm and suicide amongst children and young people: A practice focused literature review. Available at: http://www.knowsleyscb.org.uk

Gilbert, P. (2005) (Ed) Compassion: Conceptualisations, research and use in psychotherapy. Routledge. London and New York.

Grant M and Booth A. (2009) A typology of reviews: an analysis of 14 review types and associated methodologies. Health Information and Libraries Journal, 26, 91-108.

Hartnett, T. (2010). Consensus-oriented decision-making: the codm model for facilitating groups to widespread agreement. Canada: New society publishers.

Hawton K., Saunders K., O'Connor R. (2012) Self-harm and suicide in adolescents. The Lancet, Volume 379, (9834), 2373-2382

Holford R. (2011) Knowsley Health \& Wellbeing: Improving People's Lives. Suicides and Self Harm. Knowsley Council, NHS Knowsley.

Hudson B. (2013) Competition and Collaboration in the 'New NHS'. Centre for Health and Public Interest. Accessed at: http://chpi.org.uk/

Imaginative Curriculum Network (2006) Contexts for Curriculum Design: working with external pressures, LTSN Generic Centre. Available at: www.heacademy.co.uk

Littlechild B, Hawley C. (2010) Risk assessments for mental health service users: ethical, valid and reliable? Journal of Social Work, 10(2), 211-229.

Madge N. Hewitt A., Hawton K. , Jan de Wilde E., Corcoran P., Fekete S., van Heeringen K., De Leo D. and Ystgaard M. (2008) Deliberate self-harm within an international community sample of young people: comparative findings from the Child \& Adolescent Self-harm in Europe (CASE) Study. Journal of Child Psychology and Psychiatry 49(6), 667-677.

Mental Health Foundation; Camelot Foundation (2006). Truth hurts: report of the national inquiry into self-harm among young people. Mental Health Foundation: London.

Moran P., Coffey C., Romaniuk H., Olsson C., Borschmann R., Carlin J., Patton G. (2012)The natural history of self-harm from adolescence to young adulthood: a population-based cohort study. The Lancet, Volume 379 (9812), 21-27, 236-243

National CAMHS Support Service Workforce Programme/CERNIS (2011) Self-harm in Children and Young People Handbook. NCSS/CSIP: London 
National Institute for Clinical Excellence, NICE. (2011) Self Harm: Longer term management. (NICE Clinical Guideline 133). NICE: London.

National Institute for Clinical Excellence, NICE. (2004). The short-term physical and psychological management and secondary prevention of self-harm in primary and secondary care. NICE: London.

Nock M (2010) Self Injury. Annual Review of Clinical Psychology, 6(15) 1-15.

Pearson, G.A. (ed.) (2008) Why children die: a pilot study 2006: England (South West, North East and West Midlands), Wales and Northern Ireland. London: Confidential Enquiry into Maternal and Child Health [CEMACH]. (Accessed: 02 September 2014).

Ranade W., Hudson B. (2003) Conceptual Issues in Inter-Agency Collaboration. Local Government Studies, 29(3), 32-50, DOI: 10.1080/03003930308559378

Rayner, G (2012) - Shame on you: the interpersonal cycle of self-injury. (in press Issues in Mental Health Nursing)

Rayner, G., Allen, S. \& Johnson, M. (2005) "Countertransference and self-injury: A cognitive behavioural cycle." Journal of AdvancedNursing 50(1), 12-19.

Royal College of Psychiatry (2010) Self Harm, Suicide and Risk. Report CR158. London: Royal College Psychiatry

Skegg K. (2005) Self Harm. Lancet; 366: 1471-83.

Walsh B. (2007) Clinical assessment of self-injury: a practical guide. J. Clin. Psychol. 63(11):1057-1068.

Wolpert M, Fuggle P., Cottrell D., Fonagy P., Phillips J., Pilling S. Stein S., Target M. (2006) Drawing on the Evidence: Advice for Mental Health Professionals working with Children and Adolescents. $2^{\text {nd }}$ Edition. CAMHS Publications: London. 